\title{
Model-independent analysis of the Fermilab Tevatron turn-by-turn beam position monitor measurements
}

\author{
A. V. Petrenko* \\ Budker Institute of Nuclear Physics, 11 prospect Lavrentieva, Novosibirsk 630090, Russia
}

A. A. Valishev and V. A. Lebedev

Fermi National Accelerator Laboratory, P.O. Box 500, Batavia, Illinois 60510, USA

(Received 29 July 2010; published 6 September 2011)

\begin{abstract}
Coherent transverse beam oscillations in the Tevatron were analyzed with the model-independent analysis (MIA) technique. This allowed one to obtain the model-independent values of coupled betatron amplitudes, phase advances, and dispersion function around the ring from a single dipole kick measurement. In order to solve the MIA mode mixing problem which limits the accuracy of determination of the optical functions, we have developed a new technique of rotational MIA mode untangling. The basic idea is to treat each beam position monitor (BPM) as two BPMs separated in a ring by exactly one turn. This leads to a simple criterion of MIA mode separation: the betatron phase advance between any BPM and its counterpart shifted by one turn should be equal to the betatron tune and therefore should not depend on the BPM position in the ring. Furthermore, we describe a MIA-based technique to locate vibrating magnets in a storage ring.
\end{abstract}

DOI: 10.1103/PhysRevSTAB.14.092801

PACS numbers: 41.85.-p, 29.20.db, 29.85.Fj

\section{INTRODUCTION}

Turn-by-turn readings from beam position monitors (BPMs) in a storage ring are often used for accelerator optics measurements. The main advantage of this approach in comparison with alternative methods (e.g. the orbit response matrix technique [1]) is that it takes very little time to acquire turn-by-turn data. For example, one needs 2 hours of beam time to obtain the full orbit response matrix for the Fermilab Tevatron collider, compared to the $160 \mathrm{~ms}$ necessary to register 8000 turns of coherent betatron oscillations excited by a dipole kick.

The conventional harmonic analysis of turn-by-turn measurements is based on the accurate betatron tune determination [2]. In the Tevatron collider the betatron tunes are close to the linear coupling resonance and normally synchrobetatron sidebands overlap. Owing to this fact, it is difficult to use harmonic analysis for calculation of the optical functions from turn-by-turn measurements. This triggered our study of the model-independent analysis (MIA) $[3,4]$ as an alternative method. MIA is based on the correlational studies of signals from different BPMs and does not require accurate betatron tune determination.

In order to find the coupled betatron amplitudes and phase advances with MIA, it is necessary to solve the MIA mode mixing problem [4]. Previously this problem was addressed with two different approaches, namely, the

\footnotetext{
*petrenko@ngs.ru
}

Published by the American Physical Society under the terms of the Creative Commons Attribution 3.0 License. Further distribution of this work must maintain attribution to the author(s) and the published article's title, journal citation, and DOI. rotational mode untangling proposed in [4] and the independent component analysis (ICA) [5] approach based on some statistical criterion of mutual signal independence. Both these methods rely on Fourier analysis as a way to confirm that the mode separation algorithm actually works. However, due to the large chromatic tune spread and synchrobetatron sideband overlapping in the case of the Tevatron, it is often difficult to determine the quality of MIA mode separation from the Fourier transformations of the modes. In this paper we describe a new method of MIA mode untangling which does not rely on the Fourier spectrum information. Furthermore, we describe a MIA-based technique to locate vibrating magnets in a storage ring.

The paper is organized as follows. In Sec. II we outline the basics of MIA with some motivational considerations based on the particle tracking simulations. Section III presents the results of MIA applied to the Tevatron turnby-turn measurements. In Sec. IV we define the coupled betatron functions and determine their relation to MIA modes. Section V describes a new method of untangling the mixed MIA modes with applications to the Tevatron turn-by-turn measurements and simulation. Here we also compare our approach with other techniques. Section VI describes our MIA-based method to find the locations of vibrating quadrupoles in the Tevatron. Finally, Sec. VII presents conclusions.

\section{MODEL-INDEPENDENT ANALYSIS}

Let $z_{t m}$ represent horizontal or vertical beam centroid position recorded at the $m$ th monitor for the $t$ th turn. Then $B_{N \times M} \equiv\left(z_{t m}\right)$ is the beam history matrix of $N$ beam turns recorded simultaneously at $M$ monitors (each BPM signal 
is represented by some column of matrix $B$ ). The MIA is accomplished via singular value decomposition (SVD) of the beam history matrix

$$
B=U \Sigma V^{T},
$$

where $U_{N \times M}$ and $V_{M \times M}$ are orthogonal matrices (i.e., the matrices with orthonormal columns) and $\Sigma_{M \times M}=\operatorname{diag}\left(\sigma_{1}, \ldots, \sigma_{M}\right)$ is the diagonal matrix of declining singular values. Written separately for each BPM signal in the beam history matrix, Eq. (1) reads

$$
\left(\begin{array}{c}
z_{1 m} \\
\vdots \\
z_{N m}
\end{array}\right)=\sum_{j=1}^{M} \sigma_{j} v_{m j}\left(\begin{array}{c}
u_{1 j} \\
\vdots \\
u_{N j}
\end{array}\right),
$$

where $v_{m j}$ are the elements of matrix $V$ and $u_{n j}$ are the elements of matrix $U$. This sum can be truncated at some point because singular values decline rapidly. The remaining columns of matrix $U$ are the orthogonal basis of source signals also referred to as the temporal modes of MIA. For each temporal mode in $U$ there is one spatial mode represented by the column of matrix $V$. Spatial mode represents the amplitude variation along the ring for the corresponding temporal mode. Singular value with a proper index gives the overall amplitude of these two modes in the beam history matrix.

The statistical analysis described here is also called the principal component analysis (PCA) [6] and it is particularly known for its ability to compress data and reduce noise. PCA is used as a first step in numerous independent component analysis (ICA) algorithms [7], where the final signal separation is achieved according to some statistical criterion of mutual signal independence. Previously ICA was successfully applied to the turn-by-turn data analysis at the Fermilab Booster synchrotron [5]. At the end of Sec. V we compare different ICA algorithms as applied to the Tevatron.

Figure 1 shows singular values and fast Fourier transformation (FFT) amplitudes of two simulated turn-by-turn data sets for the Tevatron lattice with different sextupole settings corresponding to small and large chromaticities. In this tracking simulation the closed orbit passes through the centers of all sextupoles and the linear optics remains the same as the sextupole strength is changed. Singular value spectra in both cases are almost identical. In terms of the PCA data compression that means a good approximation exists for the beam history matrix $B$ with five temporal and five spatial modes in both cases of overlapping and nonoverlapping synchrobetatron sidebands. This property of MIA can be understood if we clarify the physical meaning of the leading MIA modes in this case.

Since the single-turn particle motion is dominated by the linear lattice properties, a good approximation for particle (and beam centroid) turn-by-turn positions can be written as

$$
\boldsymbol{X}(t, s) \approx R(s) \boldsymbol{X}_{0}(t)+\boldsymbol{D}(s) \delta(t),
$$
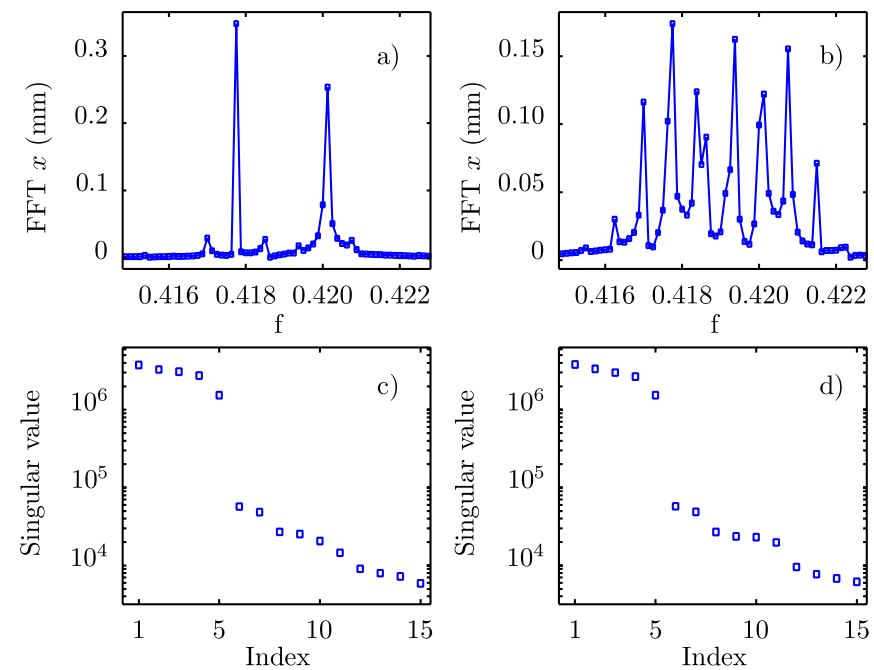

FIG. 1. Fourier amplitudes and singular values for single particle experiencing betatron and synchrotron oscillations in the Tevatron. Beam history matrix $B$ was obtained via tracking simulation in ELEGANT [19] using two different sextupole settings corresponding to small and large chromaticities. For (a) and (c) $\xi_{x}=-3, \xi_{y}=3$; for (b) and (d) $\xi_{x}=19, \xi_{y}=26$. Initial nonzero particle coordinates are $x^{\prime}=6 \times 10^{-6}$ and $\Delta p / p_{0}=4 \times 10^{-5}$.

where $\boldsymbol{X}=\left(x, p_{x}, y, p_{y}\right)^{T}$ is the vector of particle canonical coordinates in transverse phase space, $R(s)$ is the $4 \times 4$ transport matrix along the ring from location 0 to location $s, \boldsymbol{D}(s)=\left(D_{x}, D_{p_{x}}, D_{y}, D_{p_{y}}\right)^{T}$ is the vector dispersion function, and $\delta(t)=\Delta p(t) / p_{0}$ is the relative momentum deviation performing slow synchrotron oscillations (i.e., the synchrotron tune $\left.\nu_{s} \ll 1\right)$.

Although the exact form of $\boldsymbol{X}_{0}(t) \equiv \boldsymbol{X}(0, t)-\boldsymbol{D}(0) \delta(t)$ may be complicated due to accumulation of weak nonlinear effects over multiple turns, Eq. (3) means that the particle transverse coordinates $x(t, s)$ and $y(t, s)$ anywhere in the ring can be described with a linear combination of five functions $x_{0}(t), p_{x 0}(t), y_{0}(t), p_{y 0}(t)$, and $\delta(t)$. Therefore the leading temporal MIA modes in this case represent an orthonormal basis in the five-dimensional space of all linear combinations of these five functions. Corresponding spatial modes represent an orthonormal basis in the space of four linear orbits and dispersion function. Notice that the leading spatial modes are defined here only by the linear optics (up to some arbitrary rotation in the five-dimensional space).

As we can see, the structure of MIA modes near the coupling resonance is simple in spite of the complicated phase space dynamics leading to the turn-by-turn BPM signals with Fourier spectra that are difficult to interpret.

\section{MIA DECOMPOSITION RESULTS}

The Tevatron collider has 118 horizontal and 118 vertical BPMs which can simultaneously record turn-by-turn 

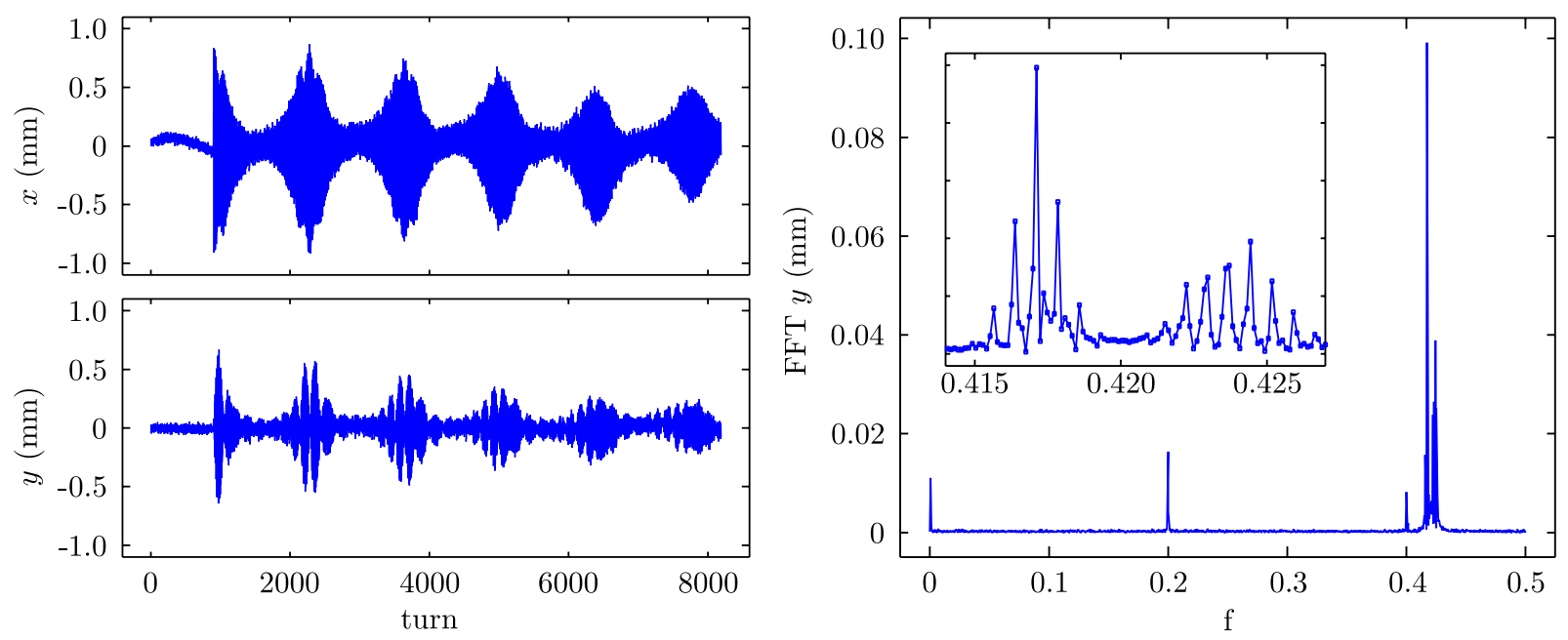

FIG. 2. Coherent transverse oscillations of proton beam in the Tevatron recorded by horizontal and vertical BPMs (left). At about 900th turn beam was kicked in the horizontal plane. FFT amplitude of the vertical BPM signal is shown on the right. Timing errors of BPM electronics with the periodicity of five turns produce coherent lines at tunes of 0.2 and 0.4 . Oscillation amplitude damping is due to nonlinear decoherence of betatron oscillations. Proton beam parameters in the Tevatron: momentum spread (rms), $1.2 \times 10^{-4}$ (at $980 \mathrm{GeV}$ ); normalized emittance (95\%), $18 \pi \mathrm{mm} \mathrm{mrad}$.

beam position histories over many thousand turns. Figure 2 shows typical turn-by-turn readings of the Tevatron BPMs when beam is kicked in the horizontal plane (full beam history matrix file is available in the Supplemental Material [8]). Figure 3 shows the MIA modes calculated from this measurement. The first four modes correspond to the betatron oscillations. The fifth mode is the lowfrequency mode which is caused by the coherent synchrotron oscillations of the beam. The spatial component of this mode is proportional to the dispersion function. The sixth mode is caused by the mechanical vibration of one of the final focus quadrupoles in the Tevatron (see Sec. VI for details). The seventh and the eighth mode is excited by timing errors of BPM electronics with periodicity of five turns resulting in coherent lines at the tunes of 0.2 and 0.4 . The spatial components of the last two modes have a typical random pattern. By looking at the spatial component of a particular mode it is easy to tell whether the mode contains some useful information about the beam motion or it is a "noise" signal generated by BPM electronics.

If we retain more singular values then other less significant noise signals and vibrational modes will appear. For example, the noise with the tune of 0.4 is below the selected singular value threshold in Fig. 3.

Fourier amplitudes of the temporal modes (see Fig. 4) reveal the residual "mixing" between the modes corresponding to different physical phenomena, i.e., each temporal mode contains low amplitude harmonics that are the
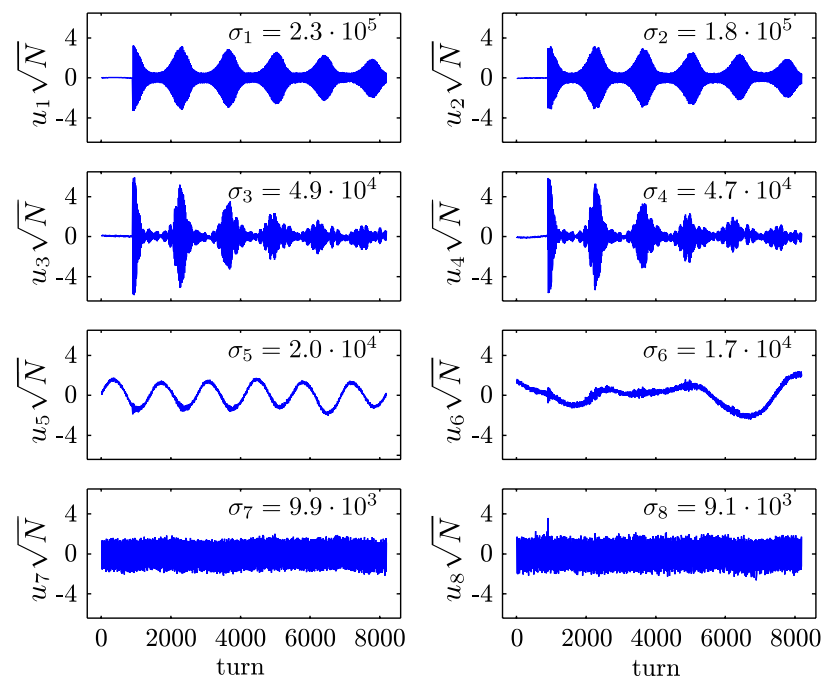
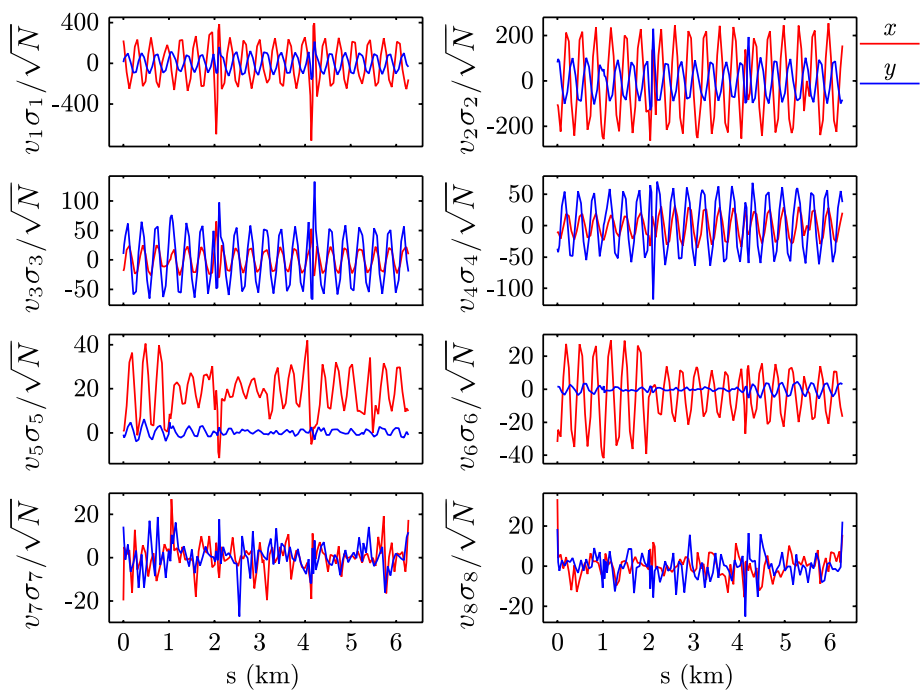

FIG. 3. Temporal (left) and spatial (right) modes of MIA corresponding to the largest eight singular values. 

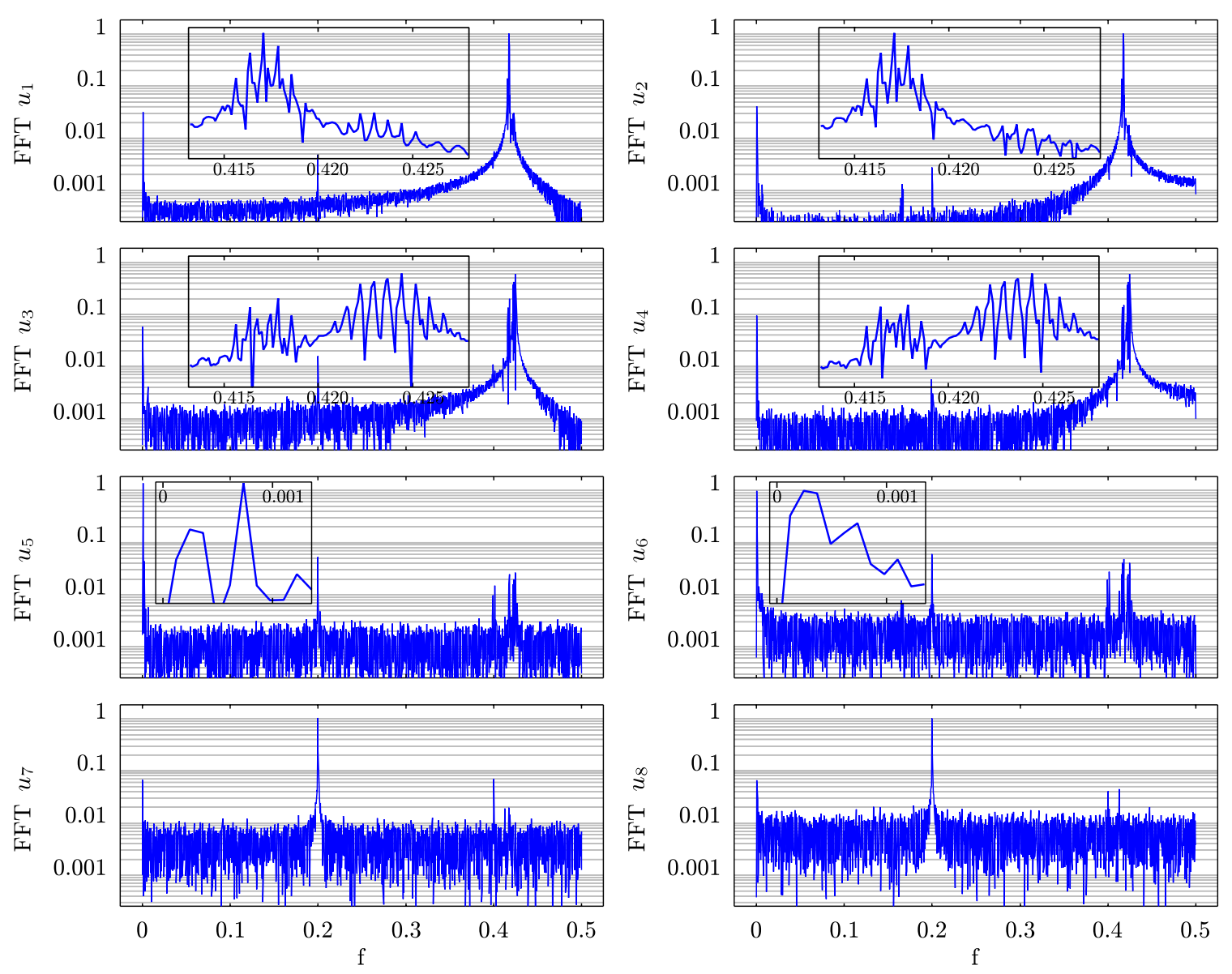

FIG. 4. FFT amplitude spectra of temporal modes presented in Fig. 3.

leading harmonics for other modes. Such mixing provides limitations for some practical applications of observed MIA modes like dispersion function measurement. There are several ways to deal with this mode mixing. First of all, one can apply a Fourier bandpass filter to data before MIA. Fourier prefiltering is useful in order to treat the lowfrequency and the high-frequency signals separately. For example, the low-pass Fourier filter makes it possible to clearly observe another vibrational mode $\left(u_{3}\right.$ in Fig. 9) in the turn-by-turn measurements presented in Fig. 2.

If the Fourier filter is not sufficient to remove the mixing between MIA modes, one can try the ICA or the general rotational mode untangling method [4]; i.e., the untangled temporal modes can be found as

$$
U_{\text {new }}=U O \text {, }
$$

where $O$ is some rotation matrix. In order to find the angles of this rotation one still needs a criterion of mode separation (Fourier amplitudes of temporal modes were used in [4]). However, in the case of overlap between synchrobetatron sidebands all these methods do not provide good betatron mode separation and simply it is hard to tell whether the betatron modes are mixed or not. Therefore we had to develop another model-independent approach which is explained in the next two sections.

\section{COUPLED BETATRON FUNCTIONS}

In this section we outline the relation between the spatial MIA modes and the optical functions that are used to describe the coupled betatron oscillations. More detailed analysis is available in [4].

Let us consider single particle betatron oscillations with linear coupling between horizontal and vertical planes. Let $L$ be the ring circumference, then $R(L)$ is the full turn transport matrix, i.e.,

$$
R(L) \boldsymbol{X}(t, 0)=\boldsymbol{X}(t+1,0) .
$$

The $R(L)$ matrix has four complex eigenvectors $\boldsymbol{\nu}_{1}, \boldsymbol{\nu}_{1}^{*}, \boldsymbol{\nu}_{2}$, $\boldsymbol{\nu}_{2}^{*}$ (where $*$ denotes complex conjugation) and four corresponding eigenvalues $e^{ \pm i \mu_{1}}, e^{ \pm i \mu_{2}}$ [9]. Any vector of initial particle coordinates $\boldsymbol{X}(0,0)$ can be represented as a linear combination of the four eigenvectors:

$$
\boldsymbol{X}(0,0)=\frac{a_{1} \boldsymbol{\nu}_{1}+a_{2} \boldsymbol{\nu}_{2}+a_{1}^{*} \boldsymbol{\nu}_{1}^{*}+a_{2}^{*} \boldsymbol{\nu}_{2}^{*}}{2} .
$$

Therefore turn-by-turn values of $\boldsymbol{X}$ are

$$
\begin{aligned}
\boldsymbol{X}(t, s) & =R(s) \boldsymbol{X}(t, 0)=R(s) R^{t}(L) \boldsymbol{X}(0,0) \\
& =\operatorname{Re}\left[a_{1} \boldsymbol{f}_{1}(s) e^{i \mu_{1} t}+a_{2} \boldsymbol{f}_{2}(s) e^{i \mu_{2} t}\right],
\end{aligned}
$$


where $\boldsymbol{f}_{1}(s)=R(s) \boldsymbol{\nu}_{1}$ and $\boldsymbol{f}_{2}(s)=R(s) \boldsymbol{\nu}_{2}$. To describe coupled betatron oscillations we introduce the betatron amplitude and phase advance functions as the phases and amplitudes of $x$ and $y$ components of these vector functions; correspondingly, for $f_{1 x}$ we have

$$
f_{1 x}(s)=\left|f_{1 x}(s)\right| e^{i \psi_{1 x}(s)} .
$$

For simplicity of notation, let us assume that initial conditions of particle motion correspond to $a_{1}=a_{2}=1$. Then the $x$ or the $y$ component of Eq. (7) reads

$$
\begin{aligned}
z(t, s)= & \left|f_{1 z}(s)\right| \cos \left[\psi_{1 z}(s)+\mu_{1} t\right] \\
& +\left|f_{2 z}(s)\right| \cos \left[\psi_{2 z}(s)+\mu_{2} t\right],
\end{aligned}
$$

or in a matrix form

$$
z(t, s)=\left(\begin{array}{c}
\cos \mu_{1} t \\
\sin \mu_{1} t \\
\cos \mu_{2} t \\
\sin \mu_{2} t
\end{array}\right)^{T}\left(\begin{array}{c}
\left|f_{1 z}(s)\right| \cos \psi_{1 z}(s) \\
-\left|f_{1 z}(s)\right| \sin \psi_{1 z}(s) \\
\left|f_{2 z}(s)\right| \cos \psi_{2 z}(s) \\
-\left|f_{2 z}(s)\right| \sin \psi_{2 z}(s)
\end{array}\right)
$$

The beam history matrix can be written as

$$
B=U_{f} V_{f}^{T} \text {, }
$$

where

$$
\begin{gathered}
U_{f}=\left(\begin{array}{cccc}
\vdots & \vdots & \vdots & \vdots \\
\cos \mu_{1} t & \sin \mu_{1} t & \cos \mu_{2} t & \sin \mu_{2} t \\
\vdots & \vdots & \vdots & \vdots
\end{array}\right), \\
V_{f}^{T}=\left(\begin{array}{ccc}
\cdots & \left|f_{1 z}\right| \cos \psi_{1 z} & \cdots \\
\cdots & -\left|f_{1 z}\right| \sin \psi_{1 z} & \cdots \\
\cdots & \left|f_{2 z}\right| \cos \psi_{2 z} & \cdots \\
\cdots & -\left|f_{2 z}\right| \sin \psi_{2 z} & \cdots
\end{array}\right) .
\end{gathered}
$$

Equation (11) is the exact solution for the beam history matrix in the case of linear single particle betatron oscillations. According to Eq. (3) in the general case of manyparticle nonlinear coupled betatron and synchrotron oscillations, the turn-by-turn position of beam centroid anywhere in the ring can be written as a linear combination of just five functions, i.e., the $\delta(t)$ and the four components of $\boldsymbol{X}_{0}(t)$. Using the Fourier filter it is easy to filter out the low-frequency components of BPM signals proportional to $\delta(t)$ and leave only the high-frequency components given by $\boldsymbol{X}_{0}(t)$. Such filtered BPM signals are given by the $x$ or the $y$ component of $R(s) \boldsymbol{X}_{0}(t)$. Since the matrix elements $R_{i j}(s)$ and the columns of matrix $V_{f}$ are both linear orbits of the same lattice, one can always write any $R_{i j}(s)$ as a linear combination of the columns of matrix $V_{f}$ (because $V_{f}$ represents a complete basis of four linear orbits). Therefore the filtered version of matrix $B$ (labeled as $\tilde{B}$ ) can be written in the form similar to Eq. (11):

$$
\tilde{B} \approx \tilde{U}_{f} V_{f}^{T},
$$

where $\tilde{U}_{f}$ is the generalized version of the $U_{f}$ matrix for the case of many-particle beam experiencing nonlinear coupled betatron and synchrotron oscillations. The fourcolumn matrix $\tilde{U}_{f}$ is difficult to find analytically; however, one can obtain it using the particle tracking simulation data as

$$
\tilde{U}_{f} \approx \tilde{B}\left(V_{f}^{\dagger}\right)^{T},
$$

where $V_{f}^{\dagger}$ is the pseudoinverse of $V_{f}$ [i.e. Eq. (15) is the solution of Eq. (14) with respect to $\tilde{U}_{f}$ ]. Therefore we can study the properties of the $\tilde{U}_{f}$ matrix using a tracking simulation.

One important property of the $U_{f}$ matrix is that, if betatron tunes are far enough from integer, half-integer, and coupling resonances, if

$$
N\left|\mu_{1}-\mu_{2}\right| / 2 \pi \gg 1,
$$

then $U_{f}$ is proportional to the orthogonal matrix

$$
U_{f}^{T} U_{f} \approx \frac{N}{2} I_{4 \times 4} .
$$

This property (with obvious modification for $a_{1} \neq 1 \neq a_{2}$ ) is still true in the general case for the $\tilde{U}_{f}$ matrix. For example, in both simulations with different chromaticities presented in Fig. 1, the product of $\tilde{U}_{f}^{T} \tilde{U}_{f}$ yields the matrix which is very close to the expected diagonal matrix (in particular, the off-diagonal elements in this matrix are about 100 times smaller than diagonal elements).

For a large number of monitors $V_{f}^{T} V_{f}$ tends to be diagonal as well, although due to strong modulation of $\left|f_{1,2 z}(s)\right|$ near collider interaction points and because typically $N \gg M$, the diagonality of $U_{f}^{T} U_{f}$ holds much better than the diagonality of $V_{f}^{T} V_{f}$. Therefore let us orthogonalize $V_{f}$ using the SVD

$$
V_{f}=\hat{V}_{f} \Sigma_{f} O_{f}^{T} .
$$

Now Eq. (11), or similarly Eq. (14), can be rewritten in the form of SVD [see Eq. (1)]:

$$
B=\left(\sqrt{\frac{2}{N}} U_{f} O_{f}\right)\left(\sqrt{\frac{N}{2}} \Sigma_{f}\right) \hat{V}_{f}^{T} .
$$

Finally, we can relate the coupled betatron amplitudes and phase advances to the SVD of beam history matrix $B$ (or $\tilde{B}$ in the general case):

$$
\begin{aligned}
U_{f} & \approx \sqrt{\frac{N}{2}} U O_{f}^{T}, \\
V_{f} & \approx \sqrt{\frac{2}{N}} V \Sigma O_{f}^{T},
\end{aligned}
$$

where we assume that only the first four modes are retained in SVD of $B$. From the four columns of matrix $V_{f}$ one can 

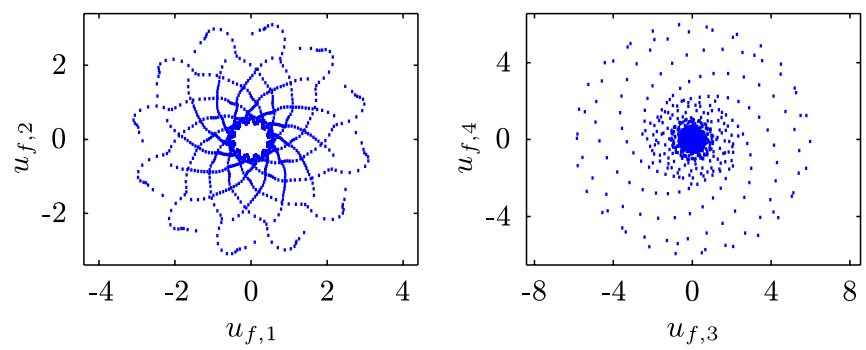

FIG. 5. Beam centroid trace in the normalized phase space (beam turn from 930 to 2100). $u_{f, 1}$ is the first column of the $\tilde{U}_{f}$ matrix, determined from the SVD of turn-by-turn measurements (see Figs. 2 and 3) using the mode untangling technique described in Sec. V.

easily calculate the coupled betatron amplitudes and phase advances. In order to obtain $V_{f}$ it is necessary to find the orthogonal $4 \times 4$ matrix $O_{f}$.

It is interesting to note that the columns of matrix $\tilde{U}_{f}$ plotted against each other show beam centroid trace in the normalized phase space (see Fig. 5).

\section{UNTANGLING BETATRON MODES}

The matrix $O_{f}$ describes some combination of rotations and reflections in four-dimensional space. Reflections only change signs of modes and can be determined if we require that the phase advance $\psi_{1 z}(s)$ and $\psi_{2 z}(s)$ calculated from $V_{f}$ increase with $s$. Any rotation in four-dimensional space can be described in terms of the six angles, each corresponding to the rotation in the plane of two basis vectors. Two of these angles are not relevant since they describe a mixing between the modes with the same tunes. This kind of rotation results simply in a phase shift, because

$$
\begin{array}{r}
\left(\begin{array}{cc}
\vdots & \vdots \\
\cos \mu_{1} t & \sin \mu_{1} t \\
\vdots & \vdots
\end{array}\right)\left(\begin{array}{cc}
\cos \theta & \sin \theta \\
-\sin \theta & \cos \theta
\end{array}\right) \\
=\left(\begin{array}{cc}
\vdots & \vdots \\
\cos \left(\mu_{1} t+\theta\right) & \sin \left(\mu_{1} t+\theta\right) \\
\vdots & \vdots
\end{array}\right) .
\end{array}
$$

The remaining four angles in $O_{f}$ set the mixing between modes with the different tunes, i.e., $O_{f}$ can be written as a product of the following four matrices:

$$
O_{13}=\left(\begin{array}{cccc}
\cos \theta_{13} & 0 & \sin \theta_{13} & 0 \\
0 & 1 & 0 & 0 \\
-\sin \theta_{13} & 0 & \cos \theta_{13} & 0 \\
0 & 0 & 0 & 1
\end{array}\right)
$$

$$
\begin{aligned}
O_{14} & =\left(\begin{array}{cccc}
\cos \theta_{14} & 0 & 0 & \sin \theta_{14} \\
0 & 1 & 0 & 0 \\
0 & 0 & 1 & 0 \\
-\sin \theta_{14} & 0 & 0 & \cos \theta_{14}
\end{array}\right), \\
O_{23} & =\left(\begin{array}{ccccc}
1 & 0 & 0 & 0 \\
0 & \cos \theta_{23} & \sin \theta_{23} & 0 \\
0 & -\sin \theta_{23} & \cos \theta_{23} & 0 \\
0 & 0 & 0 & 1
\end{array}\right), \\
O_{24} & =\left(\begin{array}{cccc}
1 & 0 & 0 & 0 \\
0 & \cos \theta_{24} & 0 & \sin \theta_{24} \\
0 & 0 & 1 & 0 \\
0 & -\sin \theta_{24} & 0 & \cos \theta_{24}
\end{array}\right) .
\end{aligned}
$$

As it was proposed in [4], one can search for these four angles by looking at Fourier amplitudes of the columns of matrix $U_{f}$ given by Eq. (20). In the case of linear betatron oscillations, the Fourier spectrum of each column in $U_{f}$ has a single peak at one of the two tunes $\mu_{1}$ or $\mu_{2}$. This approach does not work well for the Tevatron turn-byturn measurements because of significant chromatic tune spread in the beam. Even if there is no overlap between synchrobetatron sidebands (like in Fig. 2 signals), the nonlinearities in betatron oscillations produce such a distortion of Fourier spectra that MIA modes have residual components which are not possible to separate completely (see the Fourier spectra of $u_{3}$ and $u_{4}$ modes in Fig. 4). Therefore looking at Fourier spectra is usually not enough for good mode separation.

We have developed another model-independent criterion of mode separation in MIA which makes use of the fact that betatron phase advance over the entire ring is equal to the betatron tune. The idea is to treat each BPM as two monitors separated in a ring exactly by one turn. Thus, we can double the number of analyzed signals in the beam history matrix $B$ :

$B=\left(\begin{array}{cccccc}z_{11} & \cdots & z_{1 M} & z_{21} & \cdots & z_{2 M} \\ \vdots & \ddots & \vdots & \vdots & \ddots & \vdots \\ z_{N-1,1} & \cdots & z_{N-1, M} & z_{N 1} & \cdots & z_{N M}\end{array}\right)$.

Note that it is not necessary to fully double the number of signals in $B$. If calculation time is the important issue, one can duplicate only a small fraction of all available monitors.

The betatron phase advance between the monitor with readout $\left(z_{1 m}, z_{2 m}, \ldots\right)^{T}$ and its counterpart shifted by one turn $\left(z_{2 m}, z_{3 m}, \ldots\right)^{T}$ does not depend on BPM index $m$ and should be equal to the corresponding tune. Indeed, if we denote the elements of $V_{f}$ as

$$
\begin{gathered}
A_{1 z}(s)=\left|f_{1 z}(s)\right| \cos \psi_{1 z}(s), \\
B_{1 z}(s)=-\left|f_{1 z}(s)\right| \sin \psi_{1 z}(s),
\end{gathered}
$$




$$
\begin{aligned}
& \bar{A}_{1 z}(s)=A_{1 z}(s+L)=\left|f_{1 z}\right| \cos \left(\psi_{1 z}+\mu_{1}\right), \\
& \bar{B}_{1 z}(s)=B_{1 z}(s+L)=-\left|f_{1 z}\right| \sin \left(\psi_{1 z}+\mu_{1}\right),
\end{aligned}
$$

then the following expressions can be calculated at each BPM, and apparently they do not depend on a BPM location $s$ :

$$
\begin{aligned}
& \frac{A_{1 z} \bar{A}_{1 z}+B_{1 z} \bar{B}_{1 z}}{A_{1 z}^{2}+B_{1 z}^{2}}=\cos \mu_{1}, \\
& \frac{B_{1 z} \bar{A}_{1 z}-A_{1 z} \bar{B}_{1 z}}{A_{1 z}^{2}+B_{1 z}^{2}}=\sin \mu_{1} .
\end{aligned}
$$

These expressions give a sensitive criterion of betatron mode separation in MIA. In particular, one may first assume that there is no mode mixing, i.e., $O_{f}=I$, and calculate betatron tunes at each BPM using the corresponding elements of matrix $\sqrt{2 / N} V \Sigma$ [see Eq. (21)]. If the resulting BPM-by-BPM tune variation is significant, then one can minimize it with a proper $O_{f}$ matrix.

In order to explain our algorithm for the mixing matrix $O_{f}$ determination, let us assume that the mixing is small, i.e., $O_{f}$ is close to the unity matrix

$$
O_{f}=O_{13} O_{14} O_{23} O_{24} \approx I+\delta O_{13}+\delta O_{14}+\delta O_{23}+\delta O_{24} \text {, }
$$

where, for example,

$$
\delta O_{13}=\left(\begin{array}{cccc}
0 & 0 & \theta_{13} & 0 \\
0 & 0 & 0 & 0 \\
-\theta_{13} & 0 & 0 & 0 \\
0 & 0 & 0 & 0
\end{array}\right) .
$$

Now we can calculate the effect of any rotation on the BPM-by-BPM tune variation given by Eqs. (32) and (33), since it can be written as

$$
\delta \mu_{1}(s)=\frac{\partial \mu_{1}}{\partial \theta_{13}} \theta_{13}+\frac{\partial \mu_{1}}{\partial \theta_{14}} \theta_{14}+\frac{\partial \mu_{1}}{\partial \theta_{23}} \theta_{23}+\frac{\partial \mu_{1}}{\partial \theta_{24}} \theta_{24} .
$$

In order to do that let us take a derivative of

$$
\tan \mu_{1}=\frac{B_{1} \bar{A}_{1}-A_{1} \bar{B}_{1}}{A_{1} \bar{A}_{1}+B_{1} \bar{B}_{1}}
$$

with respect to $\theta_{13}$. Here $A_{1}(s)$ and $B_{1}(s)$ are defined through

$$
\left(\begin{array}{cccc}
\vdots & \vdots & \vdots & \vdots \\
A_{1} & B_{1} & A_{2} & B_{2} \\
\vdots & \vdots & \vdots & \vdots
\end{array}\right)=\left(\begin{array}{cccc}
\vdots & \vdots & \vdots & \vdots \\
A_{1 z} & B_{1 z} & A_{2 z} & B_{2 z} \\
\vdots & \vdots & \vdots & \vdots
\end{array}\right) O_{13},
$$

with a similar expression giving $\bar{A}_{1}$ and $\bar{B}_{1}$. The derivative of Eq. (37) yields $\frac{\left|f_{1 z}\right|}{\left|f_{2 z}\right|} \frac{\partial \mu_{1}}{\partial \theta_{13}}=\sin \left(\psi_{1 z}+\mu_{1}\right) \cos \left(\psi_{2 z}+\mu_{2}\right)-\sin \psi_{1 z} \cos \psi_{2 z}$.

The same operation for $\theta_{24}$ gives

$\frac{\left|f_{1 z}\right|}{\left|f_{2 z}\right|} \frac{\partial \mu_{1}}{\partial \theta_{24}}=\cos \psi_{1 z} \sin \psi_{2 z}-\cos \left(\psi_{1 z}+\mu_{1}\right) \sin \left(\psi_{2 z}+\mu_{2}\right)$.

One may note that, as soon as $\Delta \mu=\mu_{2}-\mu_{1}$ is small,

$$
\frac{\partial \mu_{1}}{\partial \theta_{13}}+\frac{\partial \mu_{1}}{\partial \theta_{24}} \approx-\frac{\left|f_{2 z}\right|}{\left|f_{1 z}\right|} \cos \left(\psi_{2 z}-\psi_{1 z}\right) \Delta \mu
$$

is also small, while

$\frac{\partial \mu_{1}}{\partial \theta_{13}}-\frac{\partial \mu_{1}}{\partial \theta_{24}} \approx 2 \frac{\left|f_{2 z}\right|}{\left|f_{1 z}\right|} \cos \left(\psi_{2 z}+\psi_{1 z}+\mu_{1}\right) \sin \mu_{1}$

is approximately twice as big as each of the derivatives. A similar result can be obtained for $\theta_{14}$ and $\theta_{23}$, namely,

$\frac{\partial \mu_{1}}{\partial \theta_{14}}+\frac{\partial \mu_{1}}{\partial \theta_{23}} \approx-2 \frac{\left|f_{2 z}\right|}{\left|f_{1 z}\right|} \sin \left(\psi_{2 z}+\psi_{1 z}+\mu_{1}\right) \sin \mu_{1}$,

and

$$
\frac{\partial \mu_{1}}{\partial \theta_{14}}-\frac{\partial \mu_{1}}{\partial \theta_{23}} \approx \frac{\left|f_{2 z}\right|}{\left|f_{1 z}\right|} \sin \left(\psi_{2 z}-\psi_{1 z}\right) \Delta \mu .
$$

Therefore in order to find the $O_{f}$ matrix which minimizes the BPM-by-BPM tune variation $\delta \mu_{1,2}(s)$, it is more convenient to use the following set of basic rotation transformations instead of $O_{13}-O_{24}$

$$
\begin{aligned}
& O_{f 1}=O_{13}\left(\theta_{1}\right) O_{24}^{T}\left(\theta_{1}\right), \\
& O_{f 2}=O_{14}\left(\theta_{2}\right) O_{23}\left(\theta_{2}\right), \\
& O_{f 3}=O_{13}\left(\theta_{3}\right) O_{24}\left(\theta_{3}\right), \\
& O_{f 4}=O_{14}\left(\theta_{4}\right) O_{23}^{T}\left(\theta_{4}\right) .
\end{aligned}
$$

The $\delta \mu_{1,2}$ is much more sensitive to $O_{f 1}$ and $O_{f 2}$ than to $O_{f 3}$ and $O_{f 4}$ rotation. Typically, most BPM-by-BPM tune variation can be reduced with $O_{f}=O_{f 1} O_{f 2}$. Because $\left|f_{2 z} / f_{1 z}\right|$ and $\psi_{2 z}-\psi_{1 z}$ tend to be approximately constant all over the ring, $O_{f 3}$ and $O_{f 4}$ add a constant shift to $\delta \mu_{1,2}$ though this shift has a different value for horizontal and for vertical BPMs. Thereby after the $\theta_{1}$ and $\theta_{2}$ angles are determined by minimizing the $\delta \mu_{1,2}$ independently in each plane, then usually there is some remaining constant difference between the tunes calculated at horizontal and at vertical BPMs. This difference can be minimized with a proper selection of $\theta_{3}$ and $\theta_{4}$ angles. If the mode mixing is strong (i.e., the angles are large), then several iterations of the described procedure are required. In the case of strong 
Before rotation:

$\left\langle\mu_{1}\right\rangle / 2 \pi=0.417214, \quad \operatorname{rms}\left(\delta \mu_{1}\right) / 2 \pi=1.0 \cdot 10^{-5}$

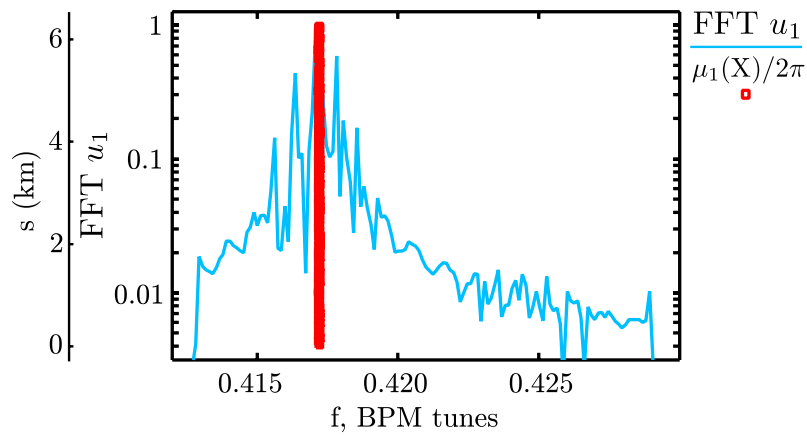

$\left\langle\mu_{2}\right\rangle / 2 \pi=0.42359, \quad \operatorname{rms}\left(\delta \mu_{2}\right) / 2 \pi=1.2 \cdot 10^{-3}$
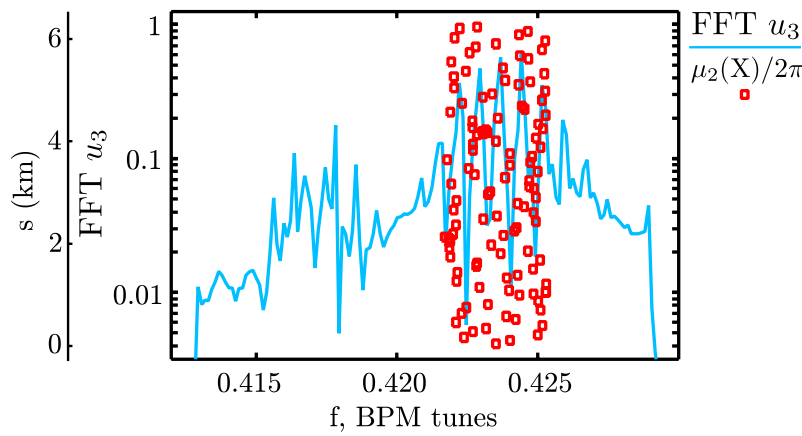

$\left\langle\mu_{1}\right\rangle / 2 \pi=0.417207, \quad \operatorname{rms}\left(\delta \mu_{1}\right) / 2 \pi=7.0 \cdot 10^{-5}$

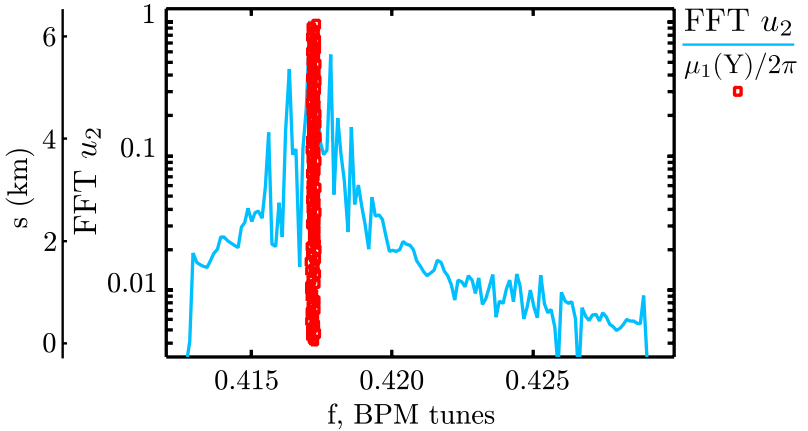

$\left\langle\mu_{2}\right\rangle / 2 \pi=0.42352, \quad \operatorname{rms}\left(\delta \mu_{2}\right) / 2 \pi=1.9 \cdot 10^{-4}$

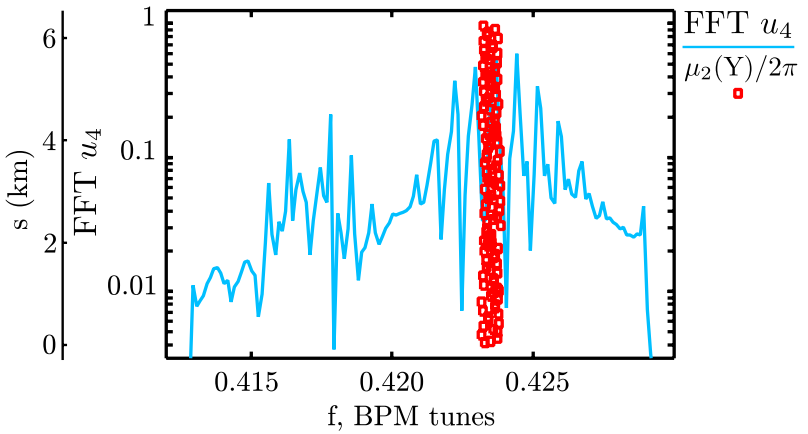

After rotation:

$\left\langle\mu_{1}\right\rangle / 2 \pi=0.417213, \quad \operatorname{rms}\left(\delta \mu_{1}\right) / 2 \pi=3.3 \cdot 10^{-6}$

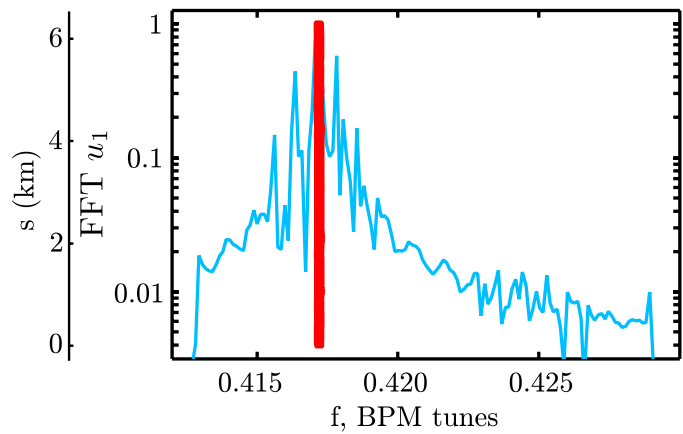

$\left\langle\mu_{2}\right\rangle / 2 \pi=0.42354, \quad \operatorname{rms}\left(\delta \mu_{2}\right) / 2 \pi=1.1 \cdot 10^{-4}$

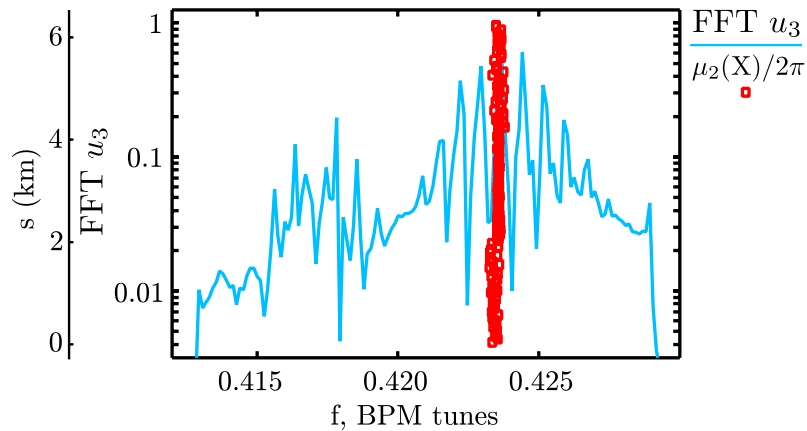

$\left\langle\mu_{1}\right\rangle / 2 \pi=0.417215, \quad \operatorname{rms}\left(\delta \mu_{1}\right) / 2 \pi=6.4 \cdot 10^{-6}$

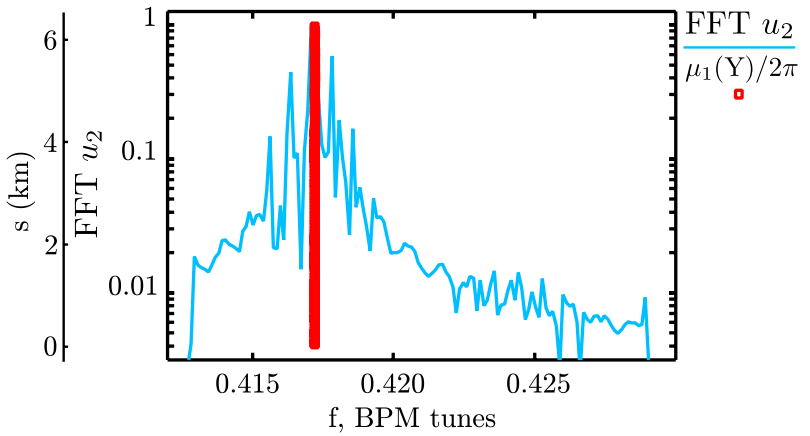

$\left\langle\mu_{2}\right\rangle / 2 \pi=0.42352, \quad \operatorname{rms}\left(\delta \mu_{2}\right) / 2 \pi=3.2 \cdot 10^{-5}$

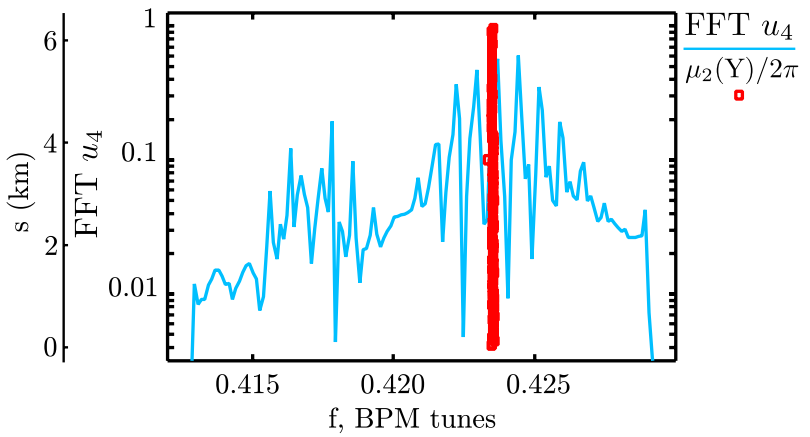

FIG. 6. Tunes calculated at each BPM using Eq. (37) and the similar one for $\mu_{2} \cdot \mu_{1}(\mathrm{X})$-betatron tune $\mu_{1}$ calculated at horizontal monitors, $\mu_{1}(\mathrm{Y})$ - the same for vertical monitors. In order to compare these results with the FFT of temporal modes (solid lines) the tunes are plotted along the horizontal $f$ axis, (vertical $s$ axis shows the positions of monitors along the ring). Fourier bandpass filter from 0.413 to 0.429 (hard edge) was applied to signals before MIA for better accuracy. 

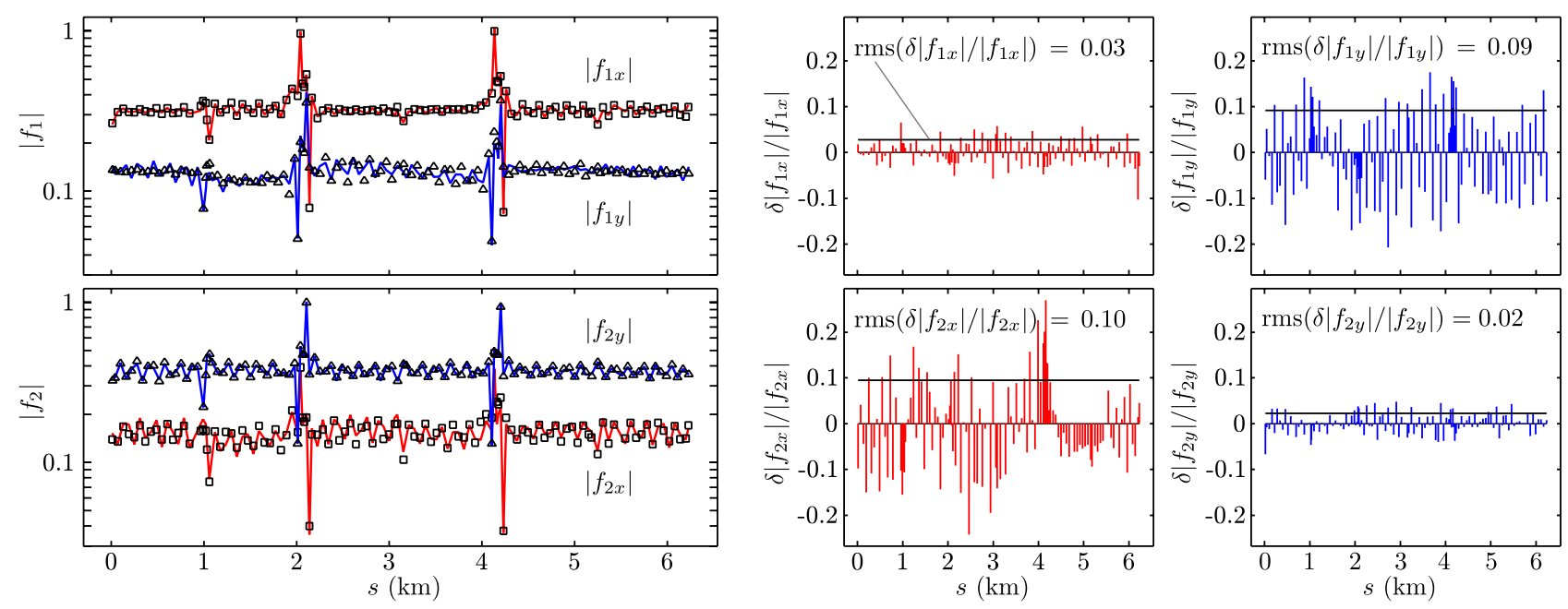

FIG. 7. Coupled betatron amplitude functions at BPMs (dots) obtained from the columns of matrix $V_{f}$ and compared to the model values (solid lines). The relative difference between the model values of optical functions and their measured values $\left(\delta\left|f_{1 x}\right|=\right.$ $\left.\left|f_{1 x}\right|_{\text {measured }}-\left|f_{1 x}\right|_{\text {model }}\right)$ is shown on the right. The same measured turn-by-turn data was used as in Fig. 2. The model was calibrated with the orbit response matrix technique [10,11]. However, since we do not know the real optics it is not clear which method (orbit response or MIA) provides the best accuracy.

mode mixing it is helpful to try several different ICA algorithms as the first step (instead of plain SVD) in the described mode untangling technique.

The smallness of $\Delta \mu$ is not a critical requirement, we employ it here just in order to explain the selection of the new set of basic rotations. The described algorithm also works for large $\Delta \mu \sim 0.1$.

Figure 6 shows the result of our method applied to untangle the betatron modes for the Tevatron turn-by-turn measurements presented in Fig. 2. As we can see from Fig. 6 in this typical case of the Tevatron turn-by-turn measurements, it is hard to determine the quality of betatron mode separation looking at the Fourier amplitudes of temporal modes. On the other hand, our new criterion provides a clear answer to this question and makes it possible to further improve the mode separation. The initial mode separation provided by the PCA in Fig. 6 is actually quite good, since the Fourier amplitudes of temporal modes before and after rotation are almost identical (although this is not a typical situation).

The coupled betatron functions calculated from the untangled modes (see Fig. 7) show good agreement with the linear model calibrated using the orbit response matrix measurements $[10,11]$. As a measure of quality of optical function determination, we will use the rms-averaged (over all BPMs) difference between the model value of optical function and its value given by the MIA. Figure 7 may serve as a typical example of how such rms average reflects the actual error distribution.

In order to benchmark our method of betatron mode untangling against some ICA algorithms, we have made the many-particle tracking simulation of Fig. 2 measurement with similar decoherence of betatron oscillations and similar chromatic tune spread in the beam. The errors of optical function determination for each algorithm applied to this simulated data are shown in Table I. Also it was possible to calculate the same optical functions using the conventional Fourier analysis [2] since in this particular case there is no overlap between the synchrobetatron sidebands corresponding to the different tunes. The MIA accuracy limit in Table I is the best possible accuracy of determination of optical functions using the four linear orbits (four spatial modes) from the SVD decomposition

TABLE I. Our method compared to several ICA algorithms and conventional harmonic analysis of turn-by-turn data. This table shows the errors of determination of betatron amplitudes via MIA. For example, $\delta\left|f_{1 x}\right|=\left|f_{1 x}\right|_{\text {MIA }}-\left|f_{1 x}\right|_{\text {model }}$ (rms average over horizontal BPMs). Very similar results are obtained for the accuracy of phase advance determination. The turn-byturn data was generated via tracking simulation with parameters similar to the measurement presented in Fig. 2.

\begin{tabular}{|c|c|c|c|c|}
\hline Method $^{\mathrm{a}}$ & $\delta\left|f_{1 x}\right| /\left|f_{1 x}\right|$ & $\delta\left|f_{1 y}\right| /\left|f_{1 y}\right|$ & $\delta\left|f_{2 x}\right| /\left|f_{2 x}\right|$ & $\delta\left|f_{2 y}\right| /\left|f_{2 y}\right|$ \\
\hline JADE & 0.0100 & 0.050 & 0.194 & 0.096 \\
\hline Plain PCA & 0.0080 & 0.041 & 0.225 & 0.044 \\
\hline Fast ICA & 0.0076 & 0.037 & 0.133 & 0.029 \\
\hline AMUSE & 0.0073 & 0.040 & 0.120 & 0.027 \\
\hline EVD & 0.0078 & 0.042 & 0.073 & 0.014 \\
\hline Our method & 0.0080 & 0.042 & 0.055 & 0.011 \\
\hline Fourier $^{\mathrm{b}}$ & 0.0077 & 0.041 & 0.050 & 0.007 \\
\hline MIA limit & 0.0005 & 0.001 & 0.005 & 0.001 \\
\hline
\end{tabular}

aMATLAB implementations of JADE, AMUSE, and EVD (see Appendix) algorithms are taken from the ICALAB package [12]. Fast ICA algorithm is from a different package [13].

${ }^{\mathrm{b}}$ In the conventional harmonic analysis of turn-by-turn data [2], we used the precise values of tunes obtained with Laskar's numerical analysis of fundamental frequencies (NAFF) [14] which is implemented in the SDDS toolkit [15]. 
of the beam history matrix $B$. In a tracking simulation this MIA accuracy limit can be easily found since we know the real lattice functions, i.e.,

$$
V_{f, \text { limit }}=V O_{\text {best }}=V V^{T} V_{f},
$$

where $O_{\text {best }}=V^{T} V_{f}$ is the best possible mode untangling matrix (note that $V V^{T} \neq I$ but $V^{T} V=I$ ). The difference between the model optics $V_{f}$ and the best possible "measured" optics $V_{f \text {,limit }}$ is caused by nonlinearities in particle motion as well as by the finite number of beam turns $N$ (which is limited by the decoherence of betatron oscillations in our case). In real measurements this limit on the MIA accuracy is also worsened by the accuracy of BPM measurements.

As can be concluded from Table I, our method has the following property when compared to the rest of ICA algorithms: it significantly improves the accuracy of one betatron function determination (i.e. $f_{2 y}$ ) at the expense of some slight increase of the errors for another function $\left(f_{1 x}\right)$.

None of the considered MIA algorithms outperforms the conventional Fourier analysis in Table I. However, the Fourier analysis is less accurate than MIA in the case of some overlap between the synchrobetatron sidebands (but when we still can find the central peaks).

In order to investigate how the separation between tunes [i.e. the Eq. (16) condition] affects the accuracy of optical

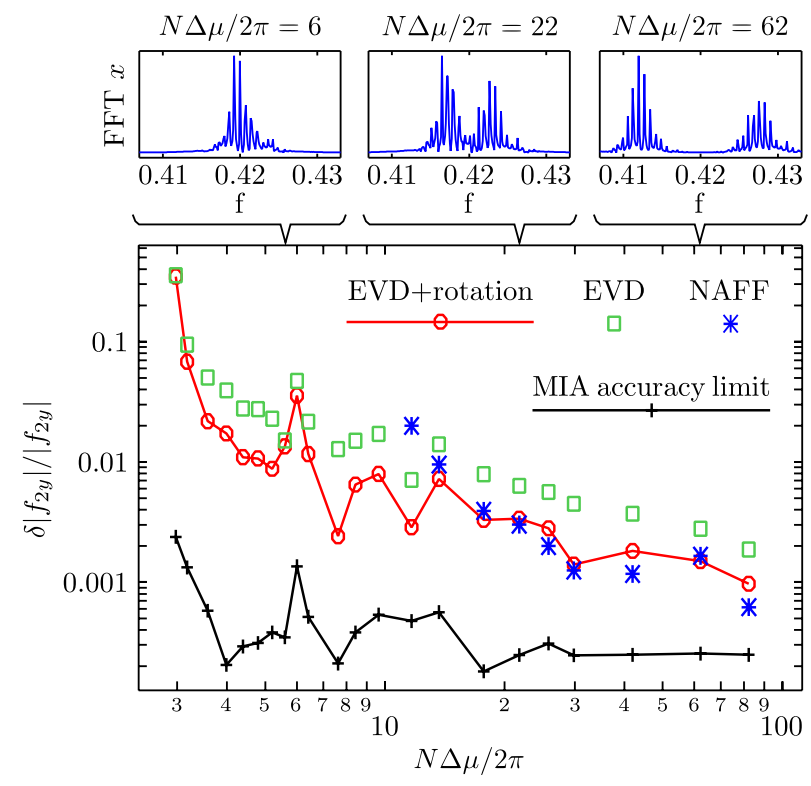

FIG. 8. Accuracy of betatron function determination versus tune separation. The turn-by-turn data was obtained via manyparticle tracking simulations with different skew-quadrupole settings. Notations are the same as in the Table I. "EVD+ rotation" means that the EVD algorithm (see Appendix) instead of plain SVD was used as initial step in our rotational mode untangling method. This approach provides the best accuracy. function measurements, we have performed another set of tracking simulations. The tune separation was regulated with skew quadrupoles, namely, in the beginning the minimum tune separation (the tune split parameter) was corrected to zero and the working point was placed close to the coupling resonance. Then using skew quadrupoles the tune split parameter was gradually increased and at each step the tracking simulation was performed providing the data for the MIA. The results of these tracking simulations are shown in Fig. 8.

\section{VIBRATING MAGNET LOCATION}

To locate the source of the vibrational mode $\left(u_{6}\right.$ in Fig. 3 or $u_{2}$ and $u_{3}$ in Fig. 9), we use an approach similar to the action and phase jump analysis [16]. Since the beam orbit in the region free of vibrating magnets is a free betatron oscillation, it can be represented as a superposition of four linearly independent orbits. The coefficients of this superposition, i.e., four initial conditions for the beam orbit, can be calculated using data from any four nearby monitors (combined function monitor is treated as two separate monitors here). These initial conditions plotted as a function of BPM sequence position along the ring should have a sudden change in the point where the beam oscillation is driven by the magnet vibration. In the case of sufficiently weak coupling, one can calculate two of the four initial conditions with any pair of nearby monitors operating in the same plane. In terms of MIA this is written as

$$
\begin{aligned}
& v_{\text {vibr }}\left(s_{1}\right)=C_{1} v_{\mathrm{b} 1}\left(s_{1}\right)+C_{2} v_{\mathrm{b} 2}\left(s_{1}\right) \\
& v_{\text {vibr }}\left(s_{2}\right)=C_{1} v_{\mathrm{b} 1}\left(s_{2}\right)+C_{2} v_{\mathrm{b} 2}\left(s_{2}\right),
\end{aligned}
$$

where $v_{\text {vibr }}$ is the spatial component of vibrational mode, $v_{\mathrm{b} 1}$ and $v_{\mathrm{b} 2}$ are the two linearly independent orbits in one plane (i.e., $v_{1}$ and $v_{2}$ modes in Fig. 3).

From Eq. (50) both initial conditions $C_{1}$ and $C_{2}$ as functions of BPM pair location in the ring can be found. It is more convenient to use the initial phase and amplitude of betatron oscillation defined as $\arctan \left(C_{1} / C_{2}\right) / 2 \pi$ and $C_{1}^{2}+C_{2}^{2}$. If there is one local source of vibrational mode,
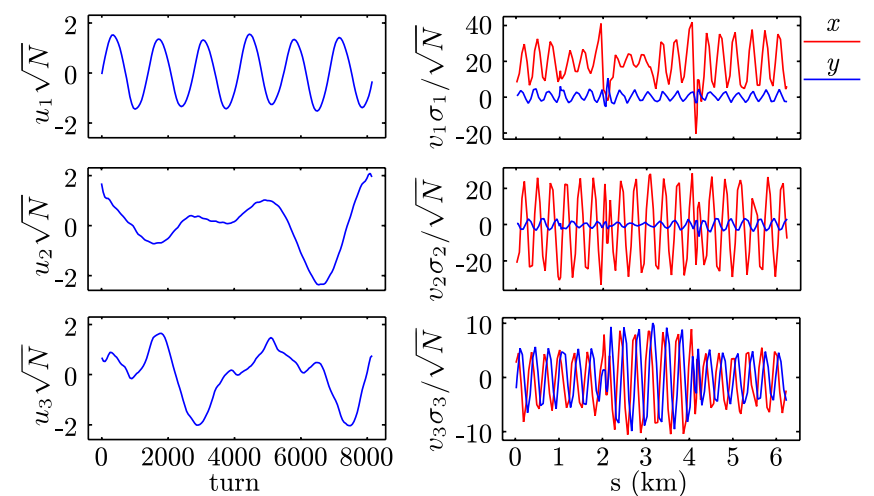

FIG. 9. Slow MIA modes. Low-pass Fourier filter $(f<0.004)$ was applied to BPM readings prior to SVD. 

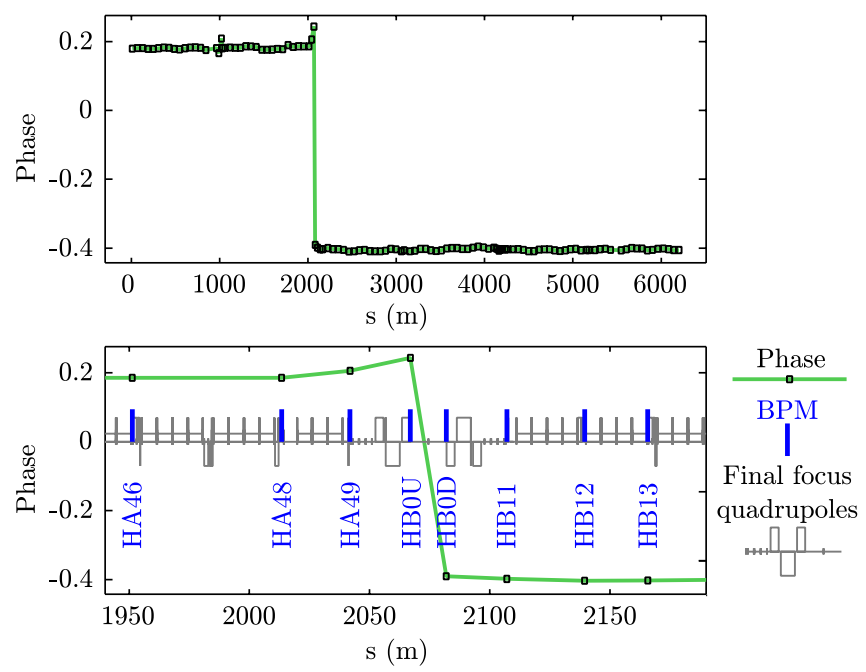

FIG. 10. Vibrating quadrupole location. The initial phase of the betatron oscillations excited by the vibrating quadrupole is shown. The phase was calculated using the pairs of horizontal monitors one after another.

then there should be a single jump of the initial phase while amplitude remains constant. Figure 10 shows the initial phase calculated for the $v_{2}$ mode in Fig. 9. As we can see this MIA mode is driven by a single local source. On the other hand, the same procedure applied to the less significant $v_{3}$ mode in Fig. 9 reveals that there are two vibrating quadrupoles located near different interaction points of the collider. The $v_{3}$ mode is the result of both these vibrations. In fact it was already evident from Fig. 9 that there are two local sources of this mode: we can see that the amplitude of the $v_{3}$ mode experience changes in two locations, while the amplitude of the $v_{2}$ mode remains constant. Note that the amplitude variation of the $v_{6}$ mode in Fig. 3 is caused by residual mode mixing. That is why the low-pass Fourier filter applied in Fig. 9 is essential for determination of vibrating quadrupole locations.

\section{CONCLUSION}

We applied model-independent analysis to the turn-byturn BPM measurements of coherent betatron oscillations excited by the transverse kicker in the Tevatron. In order to obtain the amplitudes and phase advances of coupled betatron oscillations, we have developed a new approach to rotational MIA mode untangling, which is based on the assumption that the betatron phase advance calculated between a BPM and the same BPM shifted by one turn is equal to the betatron tune and does not depend on the BPM position in the ring.

The main advantage of this method is that it can be used to untangle the mixed betatron modes in the proximity of the linear coupling resonance when there is an overlap between synchrobetatron sidebands corresponding to the different betatron tunes. However, the obvious disadvantage of our method of betatron function determination in this case is that the temporal modes are assumed to be mutually orthogonal. Therefore the fractional parts of betatron tunes cannot be too close because the condition (16) should be satisfied. This sets the lower limit on the number of turns to be recorded before the betatron oscillations in the beam decohere, namely, $N \Delta \mu / 2 \pi>4$ for typical Tevatron parameters (according to Fig. 8). One can effectively increase $N$ by treating several repeated measurements as a single BPM readout. In particular it is helpful to combine measurements with horizontal and vertical kicks.

Although according to Table I and Fig. 8 in the case of no overlap between synchrobetatron sidebands the accuracy of determination of optical functions via MIA is typically the same or worse than that of conventional Fourier analysis, our algorithm can help to find the central peak which is not always obvious in the Fourier spectrum of betatron oscillations with large chromatic tune spread as can be seen in the Fig. 2, for example.

Another application of our criterion for betatron mode separation may be the identification of malfunctioning BPMs. After the BPM-by-BPM tune spread is minimized via rotation, the tunes calculated at noisy and malfunctioning BPMs deviate from the main distribution.

We have also shown that MIA can be used to locate some unintended sources of transverse beam oscillations like the vibrating quadrupoles in the Tevatron.

So far we have used the turn-by-turn measurements only to verify the predictions of the Tevatron model which was calibrated with the orbit response matrix method. However, our final goal is to use turn-by-turn data from the Tevatron (as well as from other Fermilab accelerators) for model calibration, similarly to the technique developed at the PEPII collider $[17,18]$. The model calibration can also provide the BPM tilts and gain factors which should further improve the accuracy of optical function measurements.

\section{ACKNOWLEDGMENTS}

The authors thank Yuri Alexahin for providing many samples of the Tevatron turn-by-turn measurements. We are also grateful to Michael Borland and his APS colleagues for their excellent support of the ELEGANT program [19] and the SDDS toolkit [15]. This software was used extensively in our work.

\section{APPENDIX: EVD ALGORITHM}

We use the ICA algorithm called EVD from the ICALAB [12] package version 2.2 (the "user_alg3.m" MATLAB script written by Andrzej Cichocki and Pando Georgiev). We describe this algorithm here because the ICALAB v2.2 package is outdated and it cannot be obtained directly from the Web site [12].

The first step of EVD is the standard data whitening procedure using the SVD of covariance matrix $B^{T} B$ which 
is given by $B^{T} B=V \Sigma^{2} V^{T}$. The whitened data matrix is obtained as

$$
Z=B \tilde{V} \tilde{\Sigma}^{-1}
$$

where $\tilde{V}$ and $\tilde{\Sigma}$ are the truncated versions of $V$ and $\Sigma$, with only the largest singular values and the corresponding columns of matrix $V$ retained. Therefore in our case of coupled betatron oscillations $Z$ has four columns.

Then several time delayed covariance matrices are constructed as follows:

$$
C_{n m}(\tau)=\left\langle z_{n}(t) z_{m}(t+\tau)\right\rangle_{t}
$$

where $C_{n m}$ is the covariance matrix element, $z_{n}(t)$ is the $t$ th element of the $n$th column of matrix $Z,\langle\ldots\rangle_{t}$ stands for the averaging over turn number $t$, and $\tau$ is the time delay (we use $\tau=1,2,3,4$ ). After that the eigenvalue decomposition is used in order to diagonalize the following sum of all these time delayed covariance matrices,

$$
\sum_{\tau}\left[C(\tau)+C^{T}(\tau)\right]=W S W^{T},
$$

where $W$ is the orthogonal matrix, and $S$ is the diagonal matrix of eigenvalues. Finally, the resulting temporal modes are obtained as the columns of matrix $Z W$, and the new set of spatial modes is given by the columns of matrix $\tilde{V} \tilde{\Sigma} W$.

[1] J. Safranek, Nucl. Instrum. Methods Phys. Res., Sect. A 388, 27 (1997).

[2] J. Borer, C. Bovet, A. Burns, and G. Morpurgo, in Proceedings of the 3rd European Particle Accelerator Conference, Berlin, 1992 (Editions Frontières, Gif-surYvette, France, 1992), p. 1082.

[3] J. Irwin et al., Phys. Rev. Lett. 82, 1684 (1999).

[4] Chun-xi Wang, Phys. Rev. ST Accel. Beams 7, 114001 (2004).
[5] Xiaobiao Huang, S. Y. Lee, Eric Prebys, and Ray Tomlin, Phys. Rev. ST Accel. Beams 8, 064001 (2005).

[6] I. T. Jolliffe, Principal Component Analysis (Springer, New York, 2002).

[7] Aapo Hyvarinen, Juha Karhunen, and Erkki Oja, Independent Component Analysis (John Wiley \& Sons, New York, 2001).

[8] See Supplemental Material at http://link.aps.org/ supplemental/10.1103/PhysRevSTAB.14.092801 for the measured beam history matrix file analyzed in this article.

[9] A. Kolomensky and A. Lebedev, Theory of Cyclic Accelerators (North-Holland Publishers Company, Amsterdam, 1966).

[10] V. Sajaev et al., in Proceedings of the 21st Particle Accelerator Conference, Knoxville, 2005 (IEEE, Piscataway, NJ, 2005).

[11] V. Lebedev et al., Nucl. Instrum. Methods Phys. Res., Sect. A 558, 299 (2006).

[12] A. Cichocki et al., ICALAB Toolboxes, http:// www.bsp.brain.riken.jp/ICALAB.

[13] Hugo Gavert, Jarmo Hurri, Jaakko Sarela, and Aapo Hyvarinen, Laboratory of Information and Computer Science in the Helsinki University of Technology, http:// www.cis.hut.fi/projects/ica/fastica/.

[14] J. Laskar, C. Froeschle, and A. Celletti, Physica (Amsterdam) 56D, 253 (1992) [http://www.sciencedirect .com/science/article/pii/016727899290028L].

[15] M. Borland and L. Emery, in Proceedings of ICALEPCS 1995, Chicago, Illinois (Fermilab Report No. CONF-96/ 069), pp. 653-662, http://www.aps.anl.gov/asd/oag/software .shtml.

[16] J. F. Cardona and S. G. Peggs, Phys. Rev. ST Accel. Beams 12, 014002 (2009).

[17] Y. T. Yan and Y. Cai, Nucl. Instrum. Methods Phys. Res., Sect. A 558, 336 (2006).

[18] Y. T. Yan et al., in Proceedings of the 10th European Particle Accelerator Conference, Edinburgh, Scotland, 2006 (EPS-AG, Edinburgh, Scotland, 2006).

[19] M. Borland, Advanced Photon Source Report No. LS-287, 2000 . 